\title{
Planificación virtual y modelo de impresión en 3D para reconstrucción mandibular con colgajo libre de peroné en ameloblastoma mandibular
}

\section{Virtual planning and 3D printing model for mandibular reconstruction with free fibular flap in mandibular ameloblastoma}

Correspondencia Silvana Lucía Chang Grozo silvanachang5@hotmail.com

Recibido: 15/04/2019 Arbitrado por pares

Aprobado: 12/06/2019

Citar como: Núñez-Castañeda J, Chang-Grozo S, Izquierdo-Vela G, Iwaki-Chávez R. Planificación virtual y modelo de impresión en 3D para reconstrucción mandibular con colgajo libre de peroné en ameloblastoma mandibular. Acta Med Peru. 2019;36(3):222-6
José Nuñez-Castañeda ${ }^{1, a}$, Silvana Chang-Grozo ${ }^{1,2, b}$, Gisell Izquierdo-Vela ${ }^{1,2, b}$, Roberto Iwaki-Chávez ${ }^{1, a}$.

1 Servicio de Cirugía de Cabeza, Cuello y Máxilo-Facial, Hospital Nacional Dos de Mayo. Lima, Perú.

2 Universidad Nacional Mayor de San Marcos. Lima, Perú.

a Médico especialista en Cirugía de Cabeza, Cuello y Máxilo-Facial, b Médico Residente de Cirugía de Cabeza, Cuello y Máxilo-Facial

\section{RESUMEN}

El ameloblastoma es un tumor benigno, localmente agresivo, que ocasiona deformidades faciales y con tendencia a la recurrencia. El objetivo del tratamiento del ameloblastoma es la remoción completa del tumor, la restauración de la función y el mantenimiento del aspecto estético. Se presenta el caso de un varón de 19 años que presenta tumoración en región mandibular y acude a consulta para tratamiento quirúrgico. Se realizó planificación virtual y modelo de impresión en 3D para reconstrucción mandibular con colgajo libre de peroné y modelo estereolitográfico para el manejo de su patología. Se observó una evolución post operatoria favorable; y una patología compatible con ameloblastoma mandibular intraóseo de patrón plexiforme. Si bien la cirugía es el tratamiento de elección para el diagnóstico de ameloblastoma; la planificación virtual y el modelo de impresión en 3D para la reconstrucción son herramientas útiles en el manejo de esta patología.

Palabras clave: Ameloblastoma; Diseño asistido por computador; Estereolitografía; Colgajos tisulares libres (fuente: DeCS-BIREME).

\begin{abstract}
Ameloblastoma is a benign, locally aggressive tumor that causes facial deformities and a tendency to recur. The objective of the treatment of ameloblastoma is the complete elimination of the tumor, the restoration of function and the maintenance of the aesthetic appearance. The case of a 19-year-old male presenting a tumor in the mandibular region is presented and a consultation for surgical treatment is accessed. Virtual planning and 3D printing model were performed for mandibular reconstruction with fibula-free flap and stereolithographic model for the management of its pathology. A favorable post-operative evolution evolved; and a pathology compatible with intraosseous mandibular ameloblastoma of the plexiform pattern. Although surgery is the treatment of choice for the diagnosis of ameloblastoma; Virtual planning and the 3D printing model for reconstruction are useful tools in the management of this pathology. Keywords: Ameloblastoma; Computer-aided design; Stereolithography; Free tissue flaps (source: MeSH NLM).
\end{abstract}




\section{INTRODUCCIÓN}

El ameloblastoma es una neoplasia que se deriva del epitelio odontogénico, principalmente del esmalte no diferenciado [1], así como también de restos de lámina dental, malassez y capas de células basales del epitelio superficial suprayacente ${ }^{[2]}$. Representa aproximadamente el $1 \%$ de los tumores de cabeza y cuello y cerca del $9-11 \%$ de los tumores odontogénicos ${ }^{[3]}$.

La Organización Mundial de la Salud (OMS) lo define como una neoplasia polimórfica localmente invasiva con un patrón folicular o plexiforme dentro de un estroma fibroso ${ }^{[4]}$. El ameloblastoma se considera benigno; no obstante, tiene un comportamiento localmente agresivo, capaz de ocasionar deformidades faciales y con tendencia a la recurrencia ${ }^{[1,2]}$.

Este tumor se manifiesta entre la cuarta y quinta década de la vida; sin embargo, puede encontrarse en cualquier grupo de edad, pudiendo afectar a niños, especialmente en la variedad uniquística ${ }^{[4]}$; aunque no se ha encontrado algún tipo de predilección respecto al sexo ${ }^{[5]}$. La sintomatología es escasa, siendo la tumefacción el síntoma más común, seguida por dolor, asimetría facial y deformidad del rostro ${ }^{[2]}$.

Los objetivos del tratamiento del ameloblastoma son la remoción completa del tumor, la restauración de la función y el mantenimiento del aspecto estético ${ }^{[6]}$. Esto se logra a través de dos tipos de manejo quirúrgico: el conservador (tipo I) y el radical (tipo II). El primero incluye procedimientos como la enucleación, marsupialización, curetaje, cauterización y crioterapia ${ }^{[7]}$. En este tipo de manejo, se preserva el tejido normal del paciente, se minimiza la desfiguración facial y asegura una buena calidad de vida post-quirúrgica; no obstante, es más propenso a altas recurrencias ${ }^{[7]}$.

El segundo tipo, tratamiento quirúrgico radical, es el manejo de elección para los subtipos más agresivos y ameloblastomas recurrente. Incluye la resección del tumor en bloque con márgenes amplios de hueso, seguido de la reconstrucción del defecto óseo con injertos y rehabilitación protésica ${ }^{[7]}$. La reconstrucción mandibular sigue siendo una tarea difícil para los cirujanos de cabeza y cuello, cuyo objetivo es lograr los mejores resultados funcionales y estéticos posibles ${ }^{[8]}$.

En 1989, Hidalgo informó la utilidad de los colgajos de peroné vascularizados para la reconstrucción mandibular ${ }^{[9]}$. Desde entonces, este se ha convertido en la primera opción para la reconstrucción de la mandíbula; adicionalmente, en la actualidad, la planificación virtual y el modelado de impresión tridimensional (3D) utilizando tomografía computarizada preoperatoria se han introducido para permitir una reconstrucción más precisa ${ }^{[8]}$.

El objetivo de este reporte es describir el caso clínico de un paciente con tumor mandibular izquierdo, en el cual se utilzó la tecnología de planificación virtual y modelo de impresión en 3D para reconstrucción mandibular con colgajo libre de peroné, cuyo diagnóstico final fue ameloblastoma mandibular.

\section{REPORTE DE CASO}

Paciente varón de 19 años de edad, quien refiere que hace 03 años nota tumoración en región mandibular izquierda, no asociada a dolor ni otras molestias. Dos años después, acude a consulta odontológica, donde le solicitan una radiografía panorámica, encontrándose una imagen patológica a nivel de mandíbula izquierda (Figura 1a); motivo por el cual es referido al servicio de Cirugía de Cabeza y Cuello del Hospital Nacional Dos de Mayo (Lima - Perú).

Se le solicita tomografía espiral multicorte (TEM) de macizo facial sin contraste más reconstrucción en $3 \mathrm{D}$, describiéndose en los hallazgos una lesión osteolítica, unilocular, localizada en el cuerpo y ángulo mandibular izquierdo, comprometiendo su región alveolar y el ápex de la raíz del primer y segundo molar, que insufla y adelgaza la cortical ósea, el cual mide $36 \times 24 \times 52 \mathrm{~mm}$; hallazgos compatibles con ameloblastoma versus quíste dentígero.
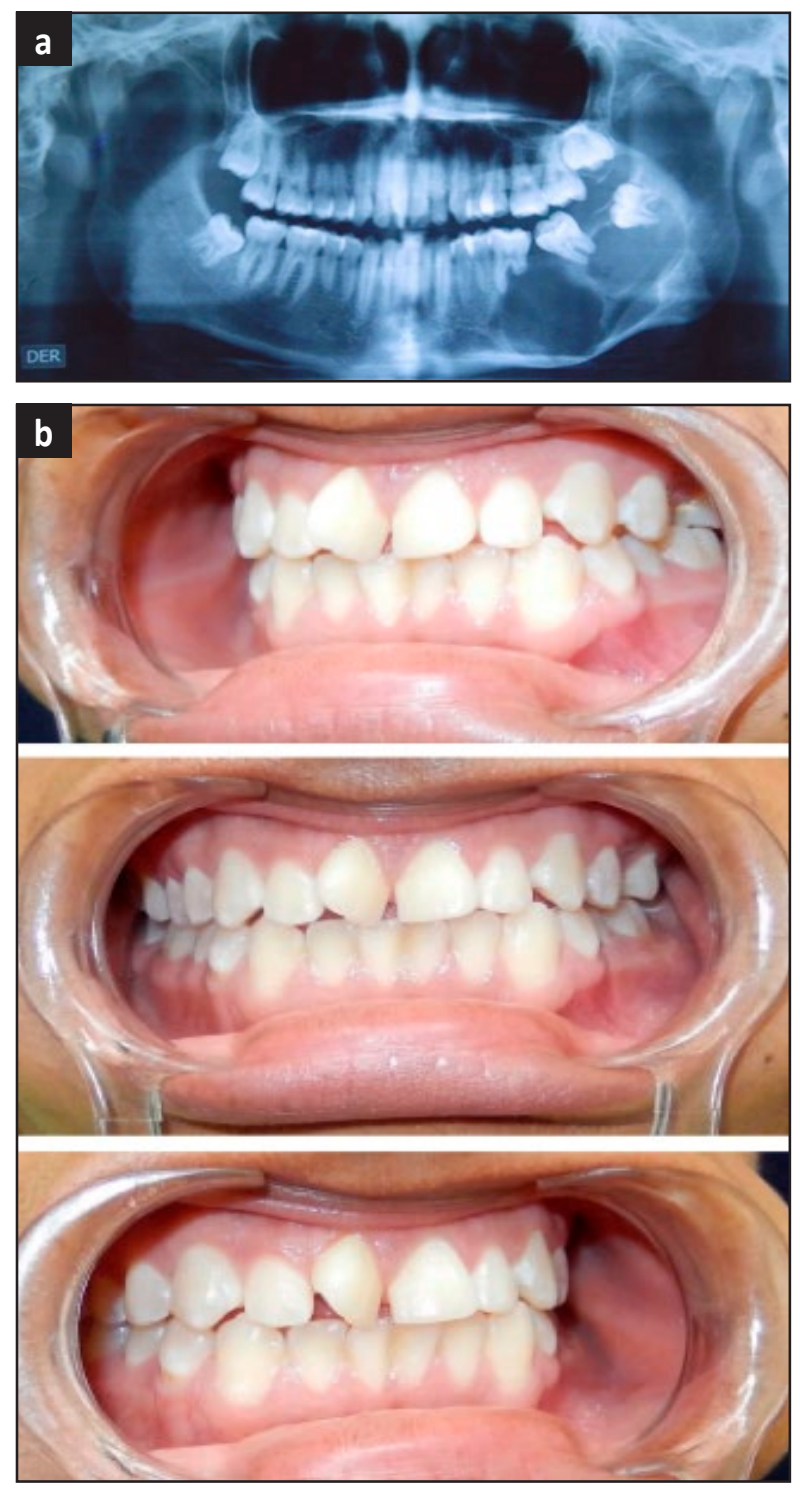

Figura 1. Evaluación pre-operatoria del paciente. a) Radiografía panorámica antes de la cirugía; b) Oclusión antes de la cirugía. 
Con estos hallazgos, se decide la intervención quirúrgica del paciente. Se empieza con la evaluación de la oclusión pretratamiento (Figura 1b) y la planificación virtual de la cirugía (Figura 2a). Así mismo, se realiza la estereolitografía de mandíbula con efecto de espejo (Figura $2 b$ ), permitiendo el doblado de la placa que será usada posteriormente en la cirugía después de su esterilización.
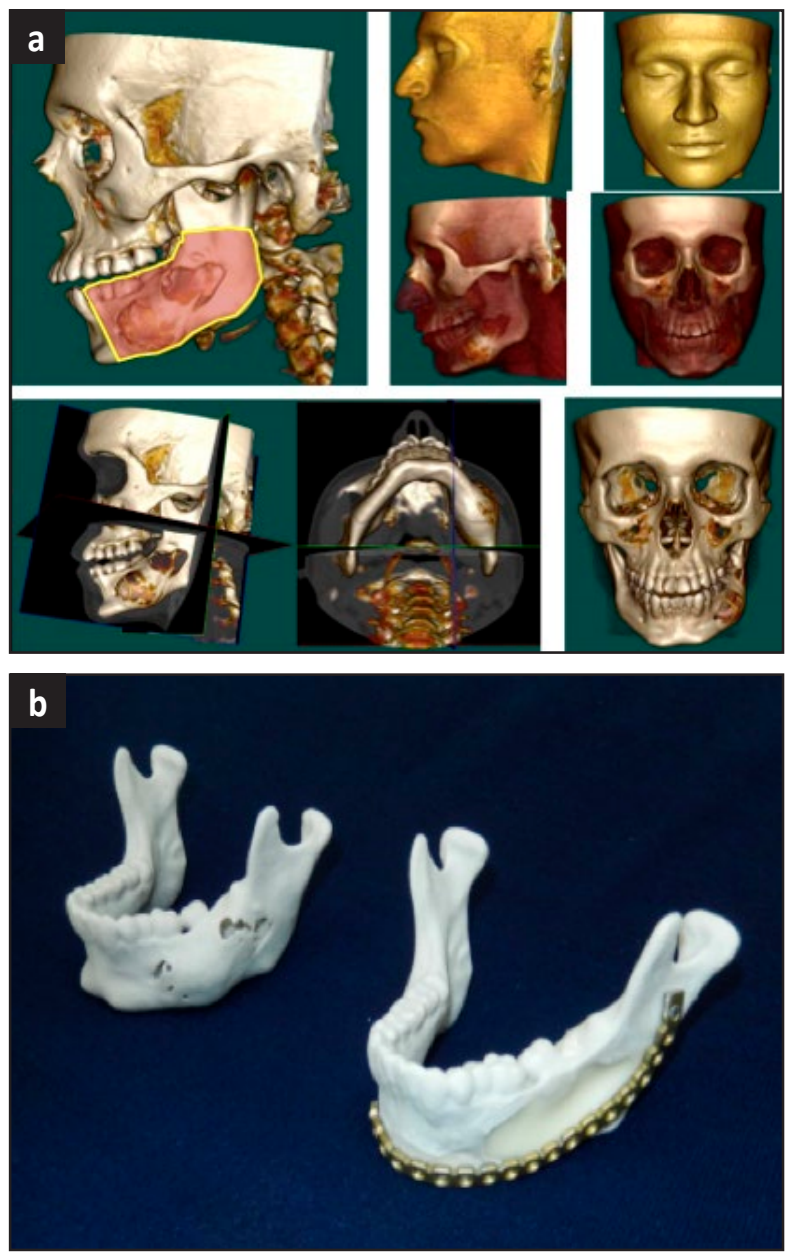

Figura 2. Planificación pre-quirúrgica. a) Planificación virtual de la cirugía; b) Estereolitografía de mandíbula con efecto de espejo, permitiendo el doblado de la placa.

La cirugía realizada fue la resección del tumor + reconstrucción mandibular con colgajo libre de peroné. En esta cirugía se emplearon dos equipos quirúrgicos simultáneos, uno de ellos a cargo de la resección del colgajo de peroné y el otro a cargo de la resección de la tumoración mandibular y anastomosis microquirúrgica. Primero, se fijó la placa preformada a nivel de peroné (Figura 3a) y se realizó la resección de hueso con sus vasos perforantes correspondientes, el cual tuvo una longitud de aproximadamente $15 \mathrm{~cm}$. Luego, se realizó la resección del tumor mandibular, el cual dejó un defecto de aproximadamente $10 \mathrm{~cm}$. Posteriormente se colocó el colgajo de peroné a nivel de cavidad oral y se realizó la unión al hueso remanente mandibular mediante osteosíntesis con tornillos de titanio a ambos extremos (Figura 3b). Si bien es cierto, como puede apreciarse en la imagen
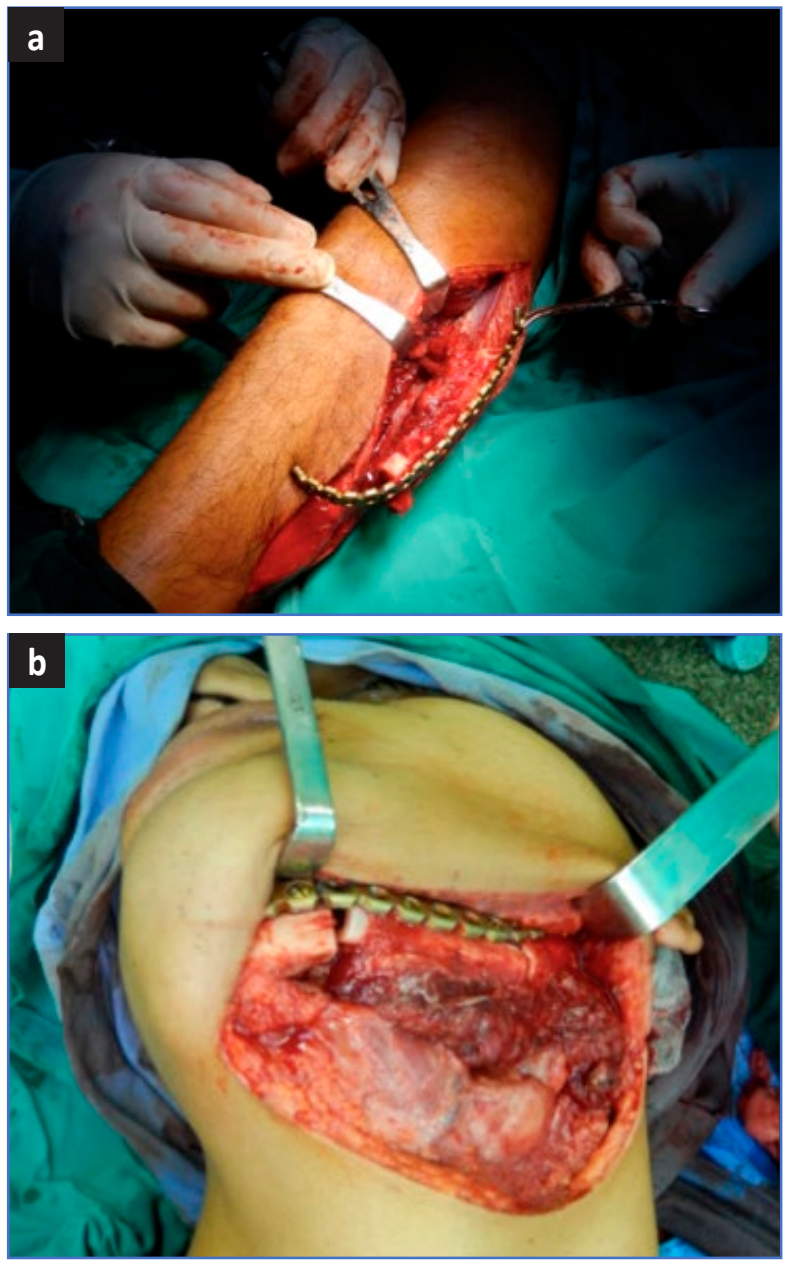

Figura 3. Momentos claves del procedimiento quirúrgico. a) Placa preformada se fija a nivel de peroné; b) Osteosíntesis con tornillos de titanio a remanente mandibular en ambos extremos.

antes mencionada, el contacto de los segmentos no es total y preciso, esto no es inconveniente para mantener la estabilidad de los fragmentos óseos, ya que este tipo de reducción con sistema de carga soportada son la placa y los tornillos los que mantienen estable el colgajo óseo.

Finalmente, se realizó microcirugía para sutura término-terminal de arteria y vena. Para esto, se uso la arteria y vena facial para la anastomosis con la arteria y vena peronea. El tiempo de cirugía fue de 06 horas. El paciente fue dado de alta a los 5 días posteriores a la cirugía, no se presentaron complicaciones ni se requirieron cirugías posteriores. Se envío la muestra mandibular al estudio anatomo-patológico, confirmándose el diagnóstico de ameloblastoma intráoseo sólido de patrón plexiforme mandibular. El paciente evolucionó favorablemente, se le realizó seguimiento mensual hasta llegar a los dos años, fecha en la cual se le tomaron radiografías control (Figura 4), en las cuales se observó el hueso peroné y las placas de titano en posición. El paciente fue evaluado posteriormente para la posibilidad de colocación de implantes dentales; no obstante, no fue candidado para ello por no contar con una adecuada altura de hueso (Figura 5). 


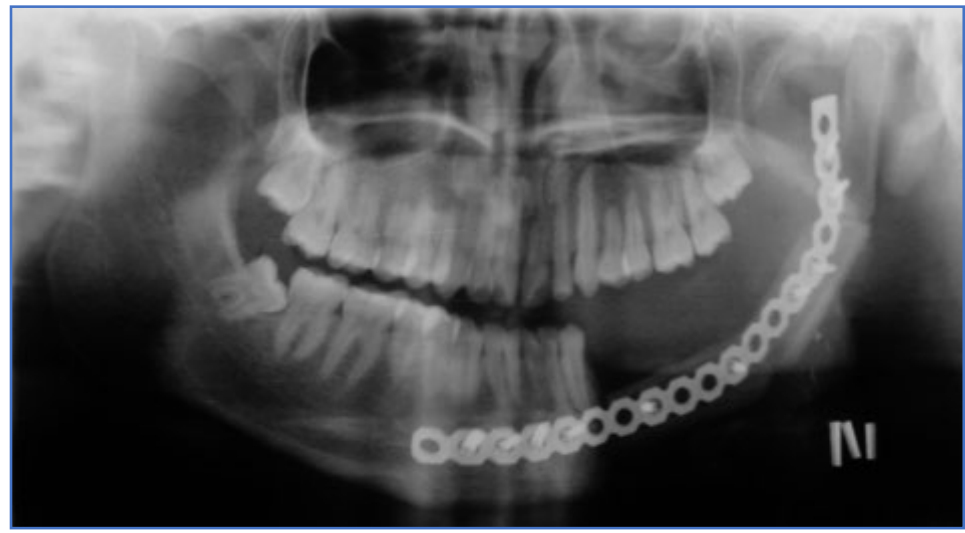

Figura 4. Radiografía control a los dos años del procedimiento quirúrgico.

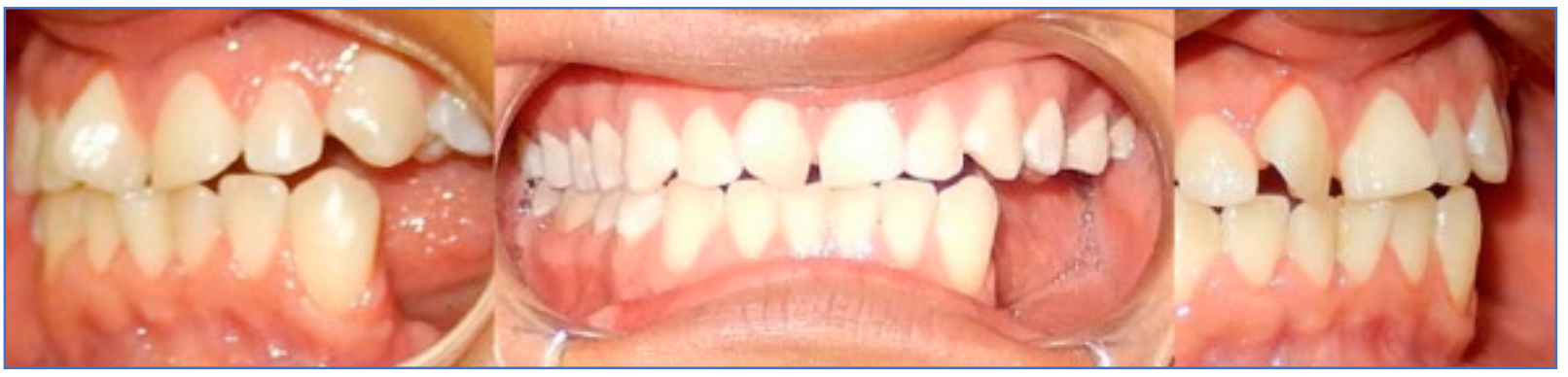

Figura 5. Oclusión post-tratamiento.

\section{DISCUSIÓN}

La actitud terapéutica del ameloblastoma es compleja, por ello la elección del tratamiento debe ser individualizada y condicionada a los factores inherentes del paciente como son: la edad, comorbilidades y posibilidad de seguimiento periódico a largo plazo ${ }^{[5,10]}$, además de las características clínicas e imagenológicas de la tumoración, como tamaño, localización y agresividad. En este caso, la lesión presentó características como compromiso de la cortical ósea y de la región alveolar del primero y segundo molar, por lo cual se optó por un tratamiento radical, el cual consiste en la resección amplia del tumor más hueso en bloque con márgenes quirúrgicos de seguridad no menores a $1 \mathrm{~cm}$ ${ }^{[3,4]}$, disminuyendo así la posibilidad de recidiva. No se realizó remoción de las partes blandas porque no hubo evidencia imagenológica de ruptura de cortical, aunque este procedimiento sólo es recomendado por algunos autores ${ }^{[4]}$.

En el plan de tratamiento se debe incluir también la reconstrucción y rehabilitación. Para este fin, se utilizó un colgajo libre microquirúrgico de peroné, el cual es el más recomendado por la disponibilidad de hasta $16 \mathrm{~cm}$ de hueso, con lo cual aporta un buen soporte para implante dental ${ }^{[11]}$. Con estas características, se puede realizar una reconstrucción completa de la mandíbula, amoldando la forma del ángulo, rama y cuerpo sin interrumpir la vascularización ósea ${ }^{[4,11]}$. Este colgajo tiene muchas ventajas, como una buena calidad de los injertos óseos bicorticales largos, el pedículo largo, el vaso ancho y la capacidad de incorporar piel y músculo ${ }^{[8]}$.
Antiguamente, el moldeado y la posición del colgajo libre de peroné en la reconstrucción mandibular se realizaban en el mismo momento de la cirugía, una vez resecado el defecto. Esto se basaba en la experiencia del cirujano y carecía de estrategias cuantitativas efectivas' lo que en ocasiones, resultaba en una oclusión y apariencia insatisfactorias ${ }^{[8]}$. Actualmente, la planificación virtual y el modelado de impresión tridimensional (3D) utilizando tomografía computarizada pre operatoria se han introducido para permitir una reconstrucción más precisa ${ }^{[12]}$. De esta manera, se puede simular la resección del hueso mandibular, el segmento y la conformación del colgajo del peroné y transferir el plan virtual a las plantillas intraoperatorias, las cuales son fáciles de obtener mediante la tecnología de impresión 3D ${ }^{[13]}$. Posteriormente, el moldeado de la placa de titanio puede realizarse en el modelo. Estas técnicas ayudan a los cirujanos a lograr una posición casi perfecta de las piezas del colgajo de peroné.

Las ventajas que otorga la planificación virtual y el modelado de impresión tridimensional frente al tratamiento convencional son la disminución del tiempo operatorio, mayor precisión en la reconstrucción, lo cual provee de mejores resultados estéticos y funcionales, como mayor simetría y satisfacción post quirúrgica por parte de los pacientes ${ }^{8}$. Zhang y colaboradores afirman que la planificación virtual para la reconstrucción mandibular disminuye el tiempo de isquemia frente al tratamiento convencional, lo cual implica menores complicaciones post operatorias ${ }^{[14]}$. Así mismo, Salles y cols. Manifiestan que se disminuye hasta un $30 \%$ del tiempo operatorio con una previa simulación del procedimiento ${ }^{[10]}$. 
Por otro lado, existen actualmente placas preformadas de acuerdo al tamaño mandibular (small, médium y large) o el sistema pre formado de placas personalizadas utilizando el sistema CAD-CAM ${ }^{[15]}$; sin embargo, ambas tecnologías no se encuentran disponibles en la actualidad en el Perú y, de existir, presentarían un muy alto costo para el paciente y el estado, en caso de instituciones públicas. El hecho de realizar el pre-formado de la placa sobre la estereolitografía (mandíbula sintética), como en el caso actual presentado, permite obtener un resultado natural con perfecta simetría y con un costo razonable.

Los ameloblastomas post operados y con márgenes adecuados, tienen un bajo índice de recurrencia y estos se detectan en un $95 \%$ durante los primeros 5 años tras la intervención inicial, y de estos un $50 \%$ durante en el primer año ${ }^{[4,10]}$. También se han descrito casos en los que la recidiva sucede más allá de este plazo. En algunas ocasiones se ha observado la transformación maligna. Por todo ello, el seguimiento del paciente a largo plazo es fundamental y se recomiendan controles clínicos y radiográficos periódicos. Tal como se evidencia en nuestro paciente, no presentó recidiva clínica ni radiográfica, pero se harán los controles anuales durante los siguientes 5 años.

Concluimos que, si bien el uso de colgajos óseos vascularizados, en general, es el gold standard para la reconstrucción mandibular; el colgajo del peroné es ideal debido a su grosor, longitud y uniformidad ósea, lo que lo convierte en el soporte idóneo para implantes y es adecuado para la cresta alveolar. No obstante, aún queda el desafío de dar forma con mayor precisión a los colgajos óseos para que la simetría facial y la función se restauren mejor y minimicen el tiempo operatorio de dicha cirugía compleja al mismo tiempo.

Por otro lado, en la práctica profesional, se puede observar que un gran porcentaje de cirugías de reconstrucción facial en nuestro país, se realizan sin planificación virtual, con la manipulación in situ de los implantes a utilizar. Esto difíficilmente puede asegurar un resultado simétrico y natural, debido a la dificultad que existe para el doblado de estas placas de reconstrucción, las cuales son de gran volumen y resistencia. Por elllo, consideramos de gran importancia que la comunidad científica tenga conocimiento de la existencia y las ventajas de esta tecnología, la cual está disponible en el mundo hace tiempo y recientemente en el Perú, con la intención de quedarse y ser cada vez más utilizada para el beneficio de nuestros pacientes.

Contribuciones de autoría: Los autores declaran haber realizado contribuciones significativas a la concepción y diseño del manuscrito, la recolección de los datos, redacción y revisión crítica del contenido del manuscrito, la aprobación final de la versión que se publicará y asumen la responsabilidad frente a todos los aspectos del manuscrito.
Fuente de financiamiento: Autofinanciado

Conflicto de intereses: Los autores declaran no tener conflicto de intereses.

\section{REFERENCIAS BIBLIOGRÁFICAS}

1. Masthan KMK, Anitha N, Krupaa J, Manikkam S. Ameloblastoma. J Pharm Bioallied Sci. 2015;7(Suppl 1):S167-170.

2. Ramos Perfecto D. Ameloblastoma. Odontol Sanmarquina. 2008;11(1):32-4.

3. Milman T, Ying G-S, Pan W, Livolsi V. Ameloblastoma: 25 Year Experience at a Single Institution. Head Neck Pathol. 2016;10(4):51320.

4. Torres Lagares D, Infante Cossío P, Hernández Guisado JM, Gutiérrez Pérez JL. Ameloblastoma mandibular: Revisión de la literatura y presentación de seis casos. Med Oral Patol Oral Cir Bucal. 2005;10(3):231-8.

5. Morales Navarro D. Ameloblastoma: Revisión de la literatura. Rev Cuba Estomatol. 2009;46(3):48-61.

6. Ruslin M, Hendra F-N, Vojdani A, Hardjosantoso D, Gazali M, Tajrin A, et al. The Epidemiology, treatment, and complication of ameloblastoma in East-Indonesia: 6 years retrospective study. Med Oral Patol Oral Cirugia Bucal. 2018;23(1):e54-8.

7. Effiom $O A$, Ogundana OM, Akinshipo AO, Akintoye SO. Ameloblastoma: current etiopathological concepts and management. Oral Dis. 2018;24(3):307-16.

8. Ren W, Gao L, Li S, Chen C, Li F, Wang Q, et al. Virtual Planning and 3D printing modeling for mandibular reconstruction with fibula free flap. Med Oral Patol Oral Cir Bucal. 2018;23(3):e359-66.

9. Hidalgo DA. Fibula free flap: a new method of mandible reconstruction. Plast Reconstr Surg. 1989;84(1):71-9.

10. Leiva N, Carranza F, Sat I. Estereolitografía en Odontología: Revisión bibliográfica. Odontol Sanmarquina. 2017;20(1):27-30.

11. Salgado CJ, Chim H, Moran S, Wei F-C, Mardini S. Fibula Flap. En: Mardini S, Wei FC, Eds. Flaps and Reconstructive Surgery. Elsevier Health Sciences; 2016. p. 596.

12. Zheng G, Su Y, Liao G, Chen Z, Wang L, Jiao P, et al. Mandible reconstruction assisted by preoperative virtual surgical simulation. Oral Surg Oral Med Oral Pathol Oral Radiol. 2012;113(5):604-11.

13. Ren WH, Gao L, Li SM, Li F, Zhi Y, Song JZ, et al. Virtual planning and 3D printing modeling for mandibular reconstruction with fibula free flap. Zhonghua Yi Xue Za Zhi. 2018;98(33):2666-70.

14. Zhang L, Liu Z, Li B, Yu H, Shen SG, Wang X. Evaluation of computerassisted mandibular reconstruction with vascularized fibular flap compared to conventional surgery. Oral Surg Oral Med Oral Pathol Oral Radiol. 2016;121(2):139-48.

15. Wilde F, Cornelius C-P, Schramm A. Computer-Assisted Mandibular Reconstruction using a Patient-Specific Reconstruction Plate Fabricated with Computer-Aided Design and Manufacturing Techniques. Craniomaxillofacial Trauma Reconstr. 2014;7(2):158-66. 